\title{
Source Localization from Time-Differences of Arrival using High-Frequency Communication Signals
}

\author{
Ehsan Zamanizadeh \\ Institute for Systems and Robotics \\ Instituto Superior Técnico \\ Av. Rovisco Pais, Torre Norte 7.10 \\ 1049-001 Lisboa, Portugal \\ Email: ezamanizadeh@isr.ist.utl.pt
}

\author{
João Gomes \\ Institute for Systems and Robotics \\ Instituto Superior Técnico \\ Av. Rovisco Pais, Torre Norte 7.22 \\ 1049-001 Lisboa, Portugal \\ Email: jpg@isr.ist.utl.pt
}

\author{
José M. Bioucas-Dias \\ Instituto de Telecomunicações \\ Instituto Superior Técnico \\ Av. Rovisco Pais, Torre Norte 10 \\ 1049-001 Lisboa, Portugal \\ Email: jose.bioucas@1x.it.pt
}

\begin{abstract}
Localization of underwater acoustic (UWA) sources using observed signals is a popular research topic that has many potential applications, both military and civilian (e.g., navigation of underwater vehicles, mine hunting, marine mammal studies). This work adopts an inverse problem framework where the temporal and spatial structure of multipath observed at an array of sensors deployed in an ocean waveguide is exploited to determine the source position. The proposed method aims at deriving useful spatial information as side information from highfrequency signals used for underwater acoustic communication. The approach involves several steps: $(i)$ estimating channel responses and segmenting wavefronts to recover the temporal and spatial structure of multipath arrivals at the receiver array; (ii) computing a coarse source position estimate with no $a$ priori knowledge of its location and only crude environmental information; (iii) refining the source location using modelbased tomographic methods that match observed vs. predicted wavefront arrival patterns across the array through ray tracing. The Coarse Source Localization (CSL) scheme uses an algorithm for free-space localization based on time differences of arrival, and several modifications are discussed to adapt it to nonhomogeneous underwater waveguides. The ensuing Model-Based Source Localization (MBSL) scheme uses an iterative linearized least-squares algorithm and benefits from the good accuracy of CSL to attain very fast convergence and avoid local extrema of its multimodal cost function. The algorithms are tested in simulation and using experimental data (CALCOM'10) for high-frequency transmissions at ranges from $200 \mathrm{~m}$ to $1 \mathrm{~km}$.
\end{abstract}

\section{INTRODUCTION}

Localizing an underwater acoustic source from signals received at an array of hydrophones has been a subject of intense research for several years, and finds many military and civilian applications. The underwater medium poses significant challenges to localization due to the complexity of sound propagation resulting from the nonhomogeneous sound speed, multiple interactions with the sea surface and bottom, and Doppler-induced compression or expansion of signals [1]. Much of the published work on underwater source localization is concerned with low-frequency sources, where interactions with poorly-characterized bottom sediment layers make for a difficult estimation problem. Matched-field processing (MFP), which uses forward computer models of sound propagation in ocean waveguides to predict pressure field values at the receiver hydrophones, has been quite sucessful at accurately and consistently localizing sources at frequencies up to several hundreds of $\mathrm{Hz}$ even in environments with strong refraction, strong bottom interactions, or markedly non-flat bathymetry [2].

There are far fewer reported experiments of source localization using high-frequency acoustic signals, on the order of 10 $\mathrm{kHz}$. One of the reasons for this is that state-of-the-art MFPtype methods are sensitive to environmental uncertainties or fluctuations, and these have an increasingly larger impact as acoustic wavelengths decrease. Yet, working at higher frequencies makes it possible to use arrays with smaller apertures, and the ambient noise level in most environments tends to be lower [3]. Moreover, operating at such high frequencies is necessary, e.g., when processing some marine mammal sounds.

Our envisioned application aims at extracting position information from received communications signals whose frequencies are on the order of tens of $\mathrm{kHz}$, where full-field inversion methods are of limited use both due to large modeling uncertainties and high required computational loads. There are several reasons why this goal, which calls for robust processing methods, might be useful in practice:

- To provide a clearer picture of the difficulties involved in a given spatial configuration of the acoustic link. This could serve as a basis for tuning the receiver architecture (e.g., the filters used in equalization) to a particular ensemble of expected channel impulse responses.

- To supply useful oceanographic information about the environment at no extra cost.

- To provide useful localization information to be incorporated into the navigation system of a vehicle in which the receiver is mounted.

In [4], [5] several interesting applications are described for the type of techniques developed here that build upon and expand the sophisticated capabilities of modern underwater modems, 
e.g., improved localization of submerged airplane black boxes, or GPS-type positioning for AUVs.

As described in the following sections, our localization criteria rely on matching observed and predicted propagation delays, in the same spirit of classical travel-time tomography, which predates full-field methods [1]. This is motivated by the common belief that it is possible to predict the delay "skeleton" of the acoustic field with reasonable accuracy at such frequencies, but the same cannot be said of pressure field values, given the extent of environmental uncertainties relative to the acoustic wavelength [3]. In this setting, common distortions that are highly disruptive of communication signals, such as extensive intersymbol interference due to multipath propagation in ocean waveguides, or Doppler compression due to motion at the receiver, transmitter, or sea surface [6] turn out to be beneficial, as they embed environmental information ("fingerprinting") into signals that can be exploited to improve the localization accuracy. For example, the physical receiver array can be expanded into a vertical stack of virtual array images associated with the various sound propagation paths, thus increasing the effective aperture and reducing the localization error.

Our high-frequency source localization approach comprises the following steps:

1) Estimation of channel responses

2) Segmentation of wavefronts

3) Coarse source localization (CSL)

4) Model-based wavefront matching (MBSL)

Steps 1-2 aim to retrieve the delay structure of multipath arrivals at the receiver array, whereas 3-4 use that information to compute source position estimates. Figure 1 provides an overview of the proposed source localization approach. The "UWA Channel Estimator" block employs $\ell_{2}-\ell_{1}$ Basis Pursuit (SpaRSA or TwIST algorithms) as a tool for estimating timevarying UWA channel responses (specifically, the parameters of delay-Doppler spread functions). By segmenting the observed impinging wavefronts from channel estimation, relative arrival time delays, with respect to the topmost hydrophone in the array, are computed. Knowing these time-differences of arrival (TDOA) and the UWA channel properties (such as hydrophone positions, sound speed profile, and bottom bathymetry) the "coarse source localization" block calculates an approximate source position in one or two iterations. The basic CSL algorithm we use is designed for free-space propagation, and we invoke it iteratively to perform fine adjustments of time delays to account for bending of rays and non-homogeneous sound speed based on the previous source estimate. These adjustments are carried out by the "ray length calculator" block in the figure. Finally, the source position is refined by the "model-based source localization" block, which uses a ray tracer as a forward model. The performances of various components shown in the figure are evaluated in simulation and using real data from the CALCOM'10 experiment conducted in Faro, Portugal, in June 2010.

The receiver array used in our experimental data (and simulations) consists of 16 hydrophones with $4 \mathrm{~m}$ spacing, vertically suspended from a buoy. The acoustic source is deployed at a depth of about $10 \mathrm{~m}$, and towed from a support ship. Source-receiver ranges vary from about $200 \mathrm{~m}$ to $1 \mathrm{~km}$. Although we only examine the localization performance for a single vertical receiver array, our methods could be used for other array configurations and for multiple arrays as well. In the latter case, localizing a source placed within the convex hull spanned by a set of deployed arrays would likely lead to better accuracy in both localization algorithms (CSL/MBSL), particularly in terms of range.

The paper is organized as follows. Section II briefly describes the $\ell_{2}-\ell_{1}$ Basis Pursuit methods used for channel response estimation. The Radon transform, Hough transform, and Fourier transform are introduced in Section III as methods for detecting and segmenting wavefronts from channel estimates. Coarse localization of UWA sources from TDOA measurements is described in Section IV. In Section V, the source position is derived by matching observed wavefronts with those predicted by an UWA channel simulator. Section VI provides numerical results on coarse and model-based source localization using both simulated and real data. Finally, Section VII outlines the main conclusions and points out directions for future research.

Notation: Superscripts $(\cdot)^{T},(\cdot)^{H}$ stand for transpose and conjugate transpose (hermitian), respectively. $\ell_{p}$ norms are denoted by $\|\cdot\|_{p}$, and $\ell_{2}$ is assumed when the argument $p$ is omitted.

\section{Channel Response Estimation}

This section briefly introduces the methods used for estimating the responses of time-varying UWA channels using $\ell_{2}-\ell_{1}$ basis pursuit. UWA channels can be considered as timevarying linear systems [6] and we characterize them by their Delay-Doppler Spread Functions (DDSF), which have many adjustable parameters but are often very sparse. Specifically, the channel model for (complex baseband) input $x(t)$ and output $y(t)$ is

$$
y(t)=\iint_{\mathbb{R}^{2}} U(\tau, \nu) x(t-\tau) e^{j 2 \pi \nu(t-\tau)} d \tau d \nu,
$$

where $U(\tau, \nu)$ denotes one value of the DDSF in the delayDoppler plane, and has the interpretation of a scatterer that creates at the receiver a replica of the transmitted signal with propagation delay $\tau$ and Doppler shift $\nu$ [7]. In practice we use a sampled version of (1).

We build upon our earlier work on sparse channel estimation through basis pursuit methods [8] to estimate such channels using a mixed $\ell_{2}-\ell_{1}$ fitting criterion. Specifically, given a portion of an observed packet (stacked in vector $\mathbf{y}$ ), whose content is known (i.e., containing training data or a marker waveform, whose samples make up a suitable regressor vector in each line of data matrix $\mathbf{X}$ ), at each receiver we solve for the vector of DDSF coefficients, $\mathbf{u}$, as follows:

$$
\min _{\mathbf{u}} \frac{1}{2}\|\mathbf{y}-\mathbf{X u}\|_{2}^{2}+\tau\|\mathbf{u}\|_{1},
$$




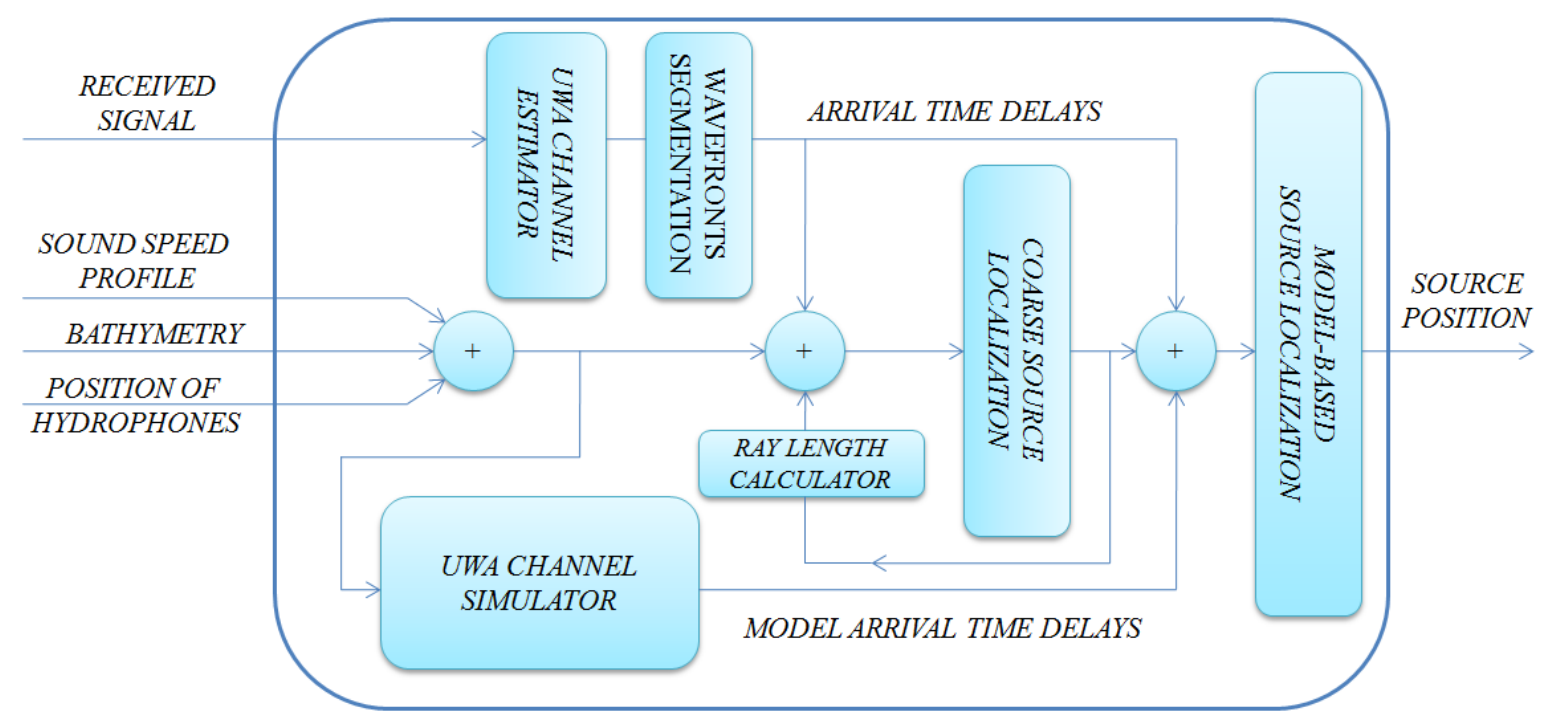

Fig. 1. Block diagram of the proposed high-frequency UWA source localization approach. Given the hydrophone positions, sound speed profile, sea floor bathymetry, and measured arrivals times of multipath components at each hydrophone, the "coarse source localization" block computes an approximate source location by solving a trilateration-type optimization problem. This approximate position will be the initial point for the "model-based source localization" block. Relative travel time delays for all the hydrophones are obtained from the observed response to a known transmission in the "UWA channel estimator" block.

The first term $\left(\ell_{2}\right)$ quantifies the least-squares (LS) fit between observed and predicted channel responses, whereas the second one $\left(\ell_{1}\right)$ is a sparsity promoting regularizer that drives to zero channel coefficients whose contribution to improve the $L S$ fit is small. The parameter $\tau$ controls the degree of sparseness in the solution. We solve (2) using the numerically efficient TwIST algorithm (Two-Step Iterative Shrinkage-Thresholding) [9] or SpaRSA (Sparse Reconstruction by Separable Approximation) [10]. For more details on the algorithms and their performance characterization see [8], [11].

\section{Segmentation of WaVefronts}

Once a sparse "skeleton" of the acoustic field is available, i.e., a sparse representation of channel coefficients as a function of delay and receiver depth, we segment it to detect wavefronts impinging upon the vertical receiver array and classify them (direct arrival, surface and bottom bounces, etc.). Our ultimate goal is to detect and classify individual multipath arrivals in estimated channel responses at each sensor for subsequent source localization steps, but we find it easier and more reliable to exploit the spatial dimension, detecting instead the distinctive delay patterns of wavefronts across the whole array.

The wavefront detection method is incoherent, given our assumption on the near-unpredictability of channel response coefficients, and consists of accumulating the energy of estimated coefficients across a set of lines in the delay-depth plane (Fig. 2). This amounts to assuming that wavefronts are planar (which is quite accurate in our experimental data, with the possible exception of the direct arrival), and performing a grid search over a candidate set of slopes and delays to locate them. Note that the plane wave approximation is only possible because a purely energetic criterion is used for detection. Even in the absence of random variations, complex amplitudes across wavefronts do not necessarily conform to a plane wave model at the operating ranges of interest.

The signatures of wavefronts could be detected across the full delay-Doppler-depth plane to retrieve not only their delay signatures but also the Doppler shifts that are related to the source-receiver relative velocity. As this would lead to high computational complexity for our approach based on grid search, we choose to project the DDSF coefficients onto the delay-depth plane, averaging $U_{m}(\tau, \nu)$ for the $m$-th sensor and delay $\tau$ across all values of the Doppler shift $\nu$. A simple parametrization for a delay-depth planar wavefront contains the angle of arrival relative to the array axis, $\phi$, and the reference delay at the topmost sensor. For a uniform linear array the candidate delay at the $m$-th sensor for this hypothetical wavefront would be

$$
\tau_{m}(\tau, \phi)=\tau+(m-1) \frac{d}{c} \sin \phi
$$

where $d$ is the intersensor separation and $c$ is the mean sound speed. Conceptually, we evaluate the wavefront energy metric for these parameters, $J(\tau, \phi)$, as

$$
J(\tau, \phi)=\sum_{m=1}^{M}\left|U_{m}\left(\tau_{m}, \cdot\right)\right| .
$$

In practice, we examine different possibilities for handling the interpolation along the delay variable that is implicit in (3)(4) when working with a discretized DDSF. In one approach we Fourier transform $U_{m}(\tau, \cdot)$ along the $\tau$ variable, replacing time-domain delaying in (4) with products by complex 
(a) Multipath profile

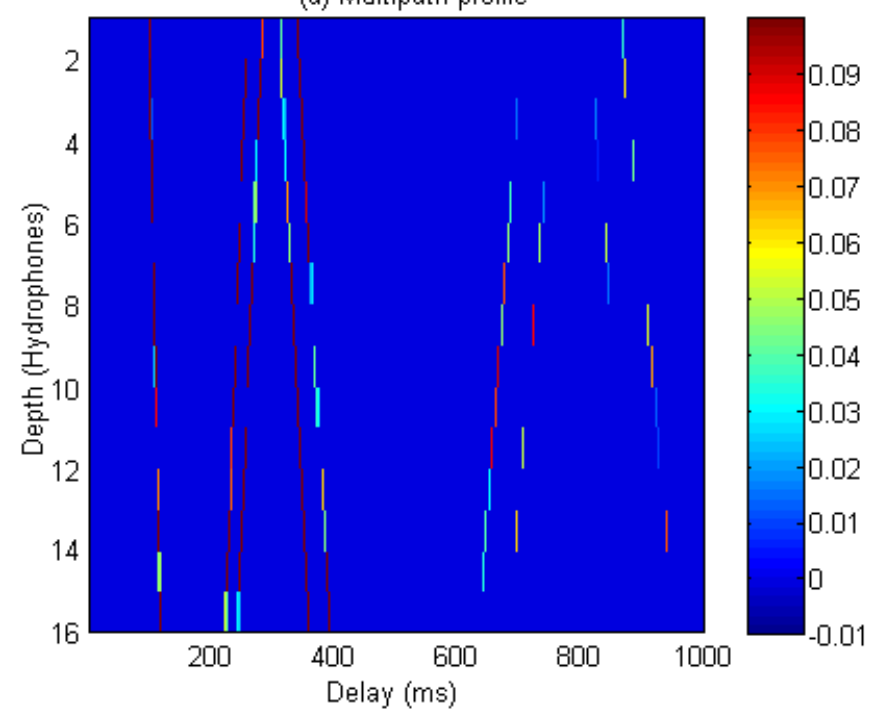

(b) Radon Transform

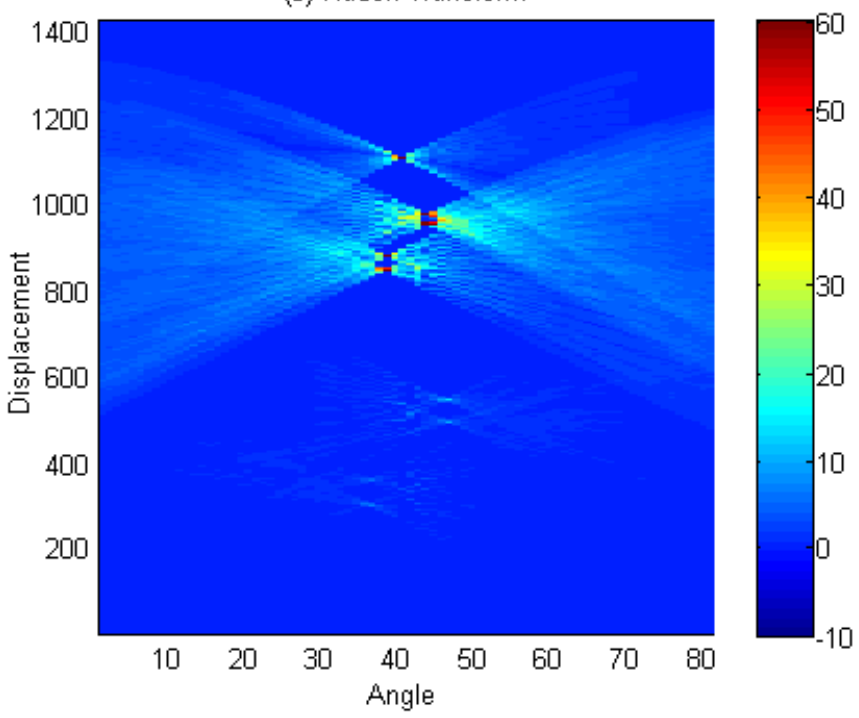

Fig. 2. (a) Arrival time delay vs. Hydrophone depth. (b) Radon transform of the top image. Each peak is related to a wavefront in the upper image.

exponentials in the frequency domain, then sum along the depth dimension and inverse Fourier transform to get $J$. This makes it simple to evaluate the metric $J$ in parallel for multiple candidate wavefronts with the same slope $\phi$ but different delays. In a similar vein, we consider alternative approaches based on the Radon and Hough transforms, which are commonly used to detect lines in images. Since the Hough Transform basically is implementable on binary images, it provides weaker results compared to those obtained by Fourier and Radon transforms.

The cost function (4) varies smoothly and contains peaks near the true set of wavefronts, but their widely-varying amplitudes preclude a simple thresholding operation to determine the wavefront parameters. We therefore resort to the following successive cancellation approach to sequentially detect wavefronts [12]:
1) Find the maximum of (4) and store the corresponding wavefront parameters.

2) Remove the effect of the detected wavefront by applying a zero mask to $U_{m}(\tau, \cdot)$ in a narrow strip along the wavefront direction/delay.

3) Recompute the energy metric for the masked DDSF and return to step 1 until the residual energy is sufficiently low.

Once labeled wavefronts are available, the corresponding delays across the array can be readily and reliably regenerated. Labelling of detected wavefronts is currently semi-automatic, and will be improved in future work.

\section{Coarse Source Localization (CSL)}

The method presented in this section provides a good initial estimate of the source position, which is needed for model-based localization when multiple maxima/minima exist in the associated cost function that matches observed vs. predicted TDOA values. The method builds upon a localization algorithm for free-space propagation based on TDOA, which we modify to include some of the effects present in ocean waveguides.

Locating a source from range measurements to known spatial reference points is a classic problem in several fields, and has received much attention in ocean applications, including active/passive sonar and beacon-aided navigation. For cooperative scenarios where source/receiver clocks are synchronous and transmit times are known, or the source operates in (receiver-initiated) transponder mode so that round-trip-times are readily available, localization can be based on absolute ranges. A common criterion is to maximize the likelihood (ML) under a Gaussian noise assumption or, equivalently,

$$
\min _{\mathbf{x}_{s}} \sum_{m=1}^{M}\left(\left\|\mathbf{x}_{s}-\mathbf{s}_{m}\right\|-d_{m}\right)^{2}
$$

where $\mathbf{x}_{s}$ and $\mathbf{s}_{m}$ denote the positions of the unknown source and the $m$-th sensor, and $d_{m}$ is the absolute distance between them [13]. In many other cases, including our experimental setup, transmit times are unknown. One possibility for localizing such sources is to match time differences of arrival between a given sensor and a reference sensor (equivalently, range differences between the sensors). In a Gaussian ML approach each term in the summation of (5) would be replaced by $\left(\left\|\mathbf{x}_{s}-\mathbf{s}_{m}\right\|-\left\|\mathbf{x}_{s}\right\|\right)-d_{m}$, where $d_{m}$ is now the measured difference between the range from the source to sensor $\mathbf{s}_{m}$, and the range from the source to the reference sensor, located at the origin of the coordinate system. The method we use is presented in [13], and matches squared range differences according to the cost function

$$
\min _{\mathbf{x}_{s}} \sum_{m=1}^{M}\left(\left\|\mathbf{x}_{s}-\mathbf{s}_{m}\right\|^{2}-\left(d_{m}+\left\|\mathbf{x}_{s}\right\|\right)^{2}\right)^{2} .
$$

By doing so, (6) may be recast as the minimization of a quadratic function subject to a quadratic constraint and a linear constraint. Surprisingly, an efficient iterative algorithm 
is proposed in [13] to find a global minimum of (6), even though, in general, this problem is not convex. This is an extremely useful property, as it eliminates issues related to convergence towards local extrema that commonly affect MLtype algorithms.

As the source localization method in [13] assumes freespace propagation, some adaptations are needed to account for the presence of multiple wavefronts in an ocean waveguide. We adopt the image method [1] to replace the physical array embedded in the waveguide with multiple virtual images in free space, associated with surface and bottom reflections, and then apply the method of [13] to determine the source position, as depicted in Fig. 3. To derive range differences from the source to the array hydrophones, the wavefront segmentation methods described in Section III are first used to estimate delay differences. Detecting wavefronts as a whole across the array provides much more robust detection and classification performance than would be possible by independently processing individual hydrophone signals. Multiplying theses time delays, $\tau_{m}$, by an average sound speed, $c_{m}$, provides distance differences to plug into (6), $d_{m}=c_{m} \tau_{m}$. We measure all delays relative to the earliest arrival (direct path) in the topmost hydrophone.

In UWA channels, due to changes in physical properties such as water temperature, density, salinity, or hydrostatic pressure, acoustic waves propagate with different speed at different depths. This non-homogeneous characteristic should be accounted for when computing range-differences for each hydrophone. Denoting by $c(z)$ the sound speed as a function of depth, its mean between the source depth, $z_{s}$, and each hydrophone at depth $z_{m}$ is a more accurate approximation to be applied in equation (6) than a global mean velocity,

$$
c_{m}=\frac{1}{z_{m}-z_{s}} \int_{z_{s}}^{z_{m}} c(z) d z .
$$

Since the source position is unknown, the CSL technique can be used iteratively to gradually refine the mean velocities in (7). In the first iteration a single global average sound speed is used to compute an initial source position, and in subsequent iterations the most recent estimate $z_{s}$ is used in (7). Similarly to the receiver array, the sound speed profile (SSP) is expanded into surface and bottom-reflected images for computing averages according to (7), with the understanding that $z_{m}$ may denote either the depth of an actual sensor or that of one of its reflected images. This is shown in Figure 4c).

\section{A. Compensating for Sloped Bathymetry in CSL}

The basic CSL method depicted in Figure 3 assumes flat (horizontal) bathymetry. In real scenarios it could have any shape, and is better approximated by piecewise-linear segments. For a single segment (constant slope) the modifications relative to the horizontal case are minor, and amount to reflecting bottom-interacting hydrophone images along the new normal, as depicted in Figure 4. For practical mild slopes the error is negligible if the sequence of reflected SSPs is still built under the assumption of flat bottom located at approximately

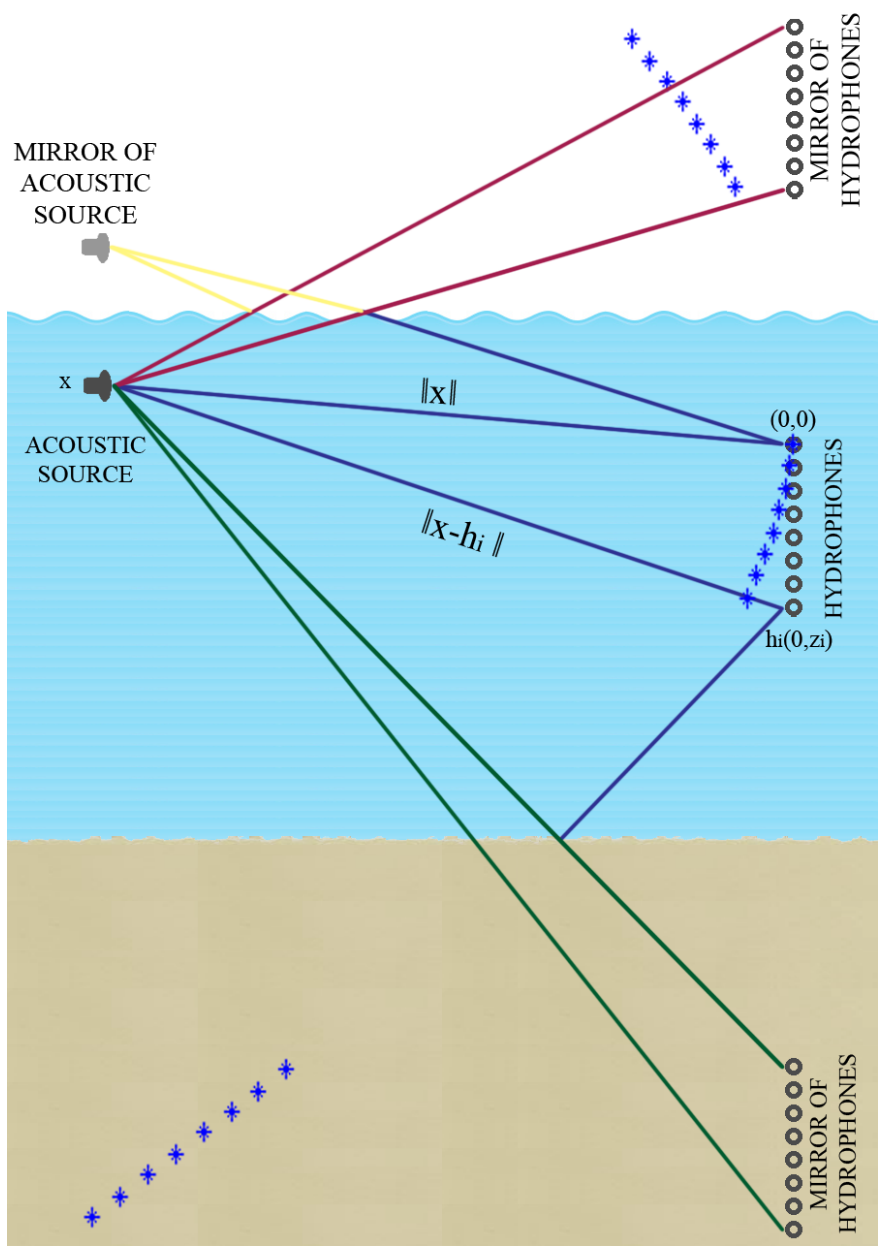

Fig. 3. Decomposition of the physical array into virtual surface and bottomreflected images. Each arrival is matched to the propagation delay between the source and the associated image hydrophone.

the average bottom depth for the operating area of interest. Note that a sloping bottom induces a non-linear expanded array shape and, in theory, endows it with the ability to resolve the left-right ambiguity for source location.

With multiple piecewise-linear bottom segments an iterative scheme is needed to determine the appropriate normal for each bottom reflection based on the current source position estimate. All the results presented in this paper approximate the bottom with a single sloped line.

\section{B. Compensating for Acoustic Ray Bending}

Acoustic rays do not travel along straight paths due to variations in sound speed, whereas the basic CSL criterion (6) is built under the assumption that travel times are proportional to Euclidean distance (i.e., straight paths, constant speed). The CSL method should account for changes in mean sound speed when translating TDOAs into range differences, as discussed in Section IV, but also for the departure of ray trajectories from the straight line assumption. For compensating either of these distortions we resort to iterative processing, using the previous source position estimate to determine the required corrections, 

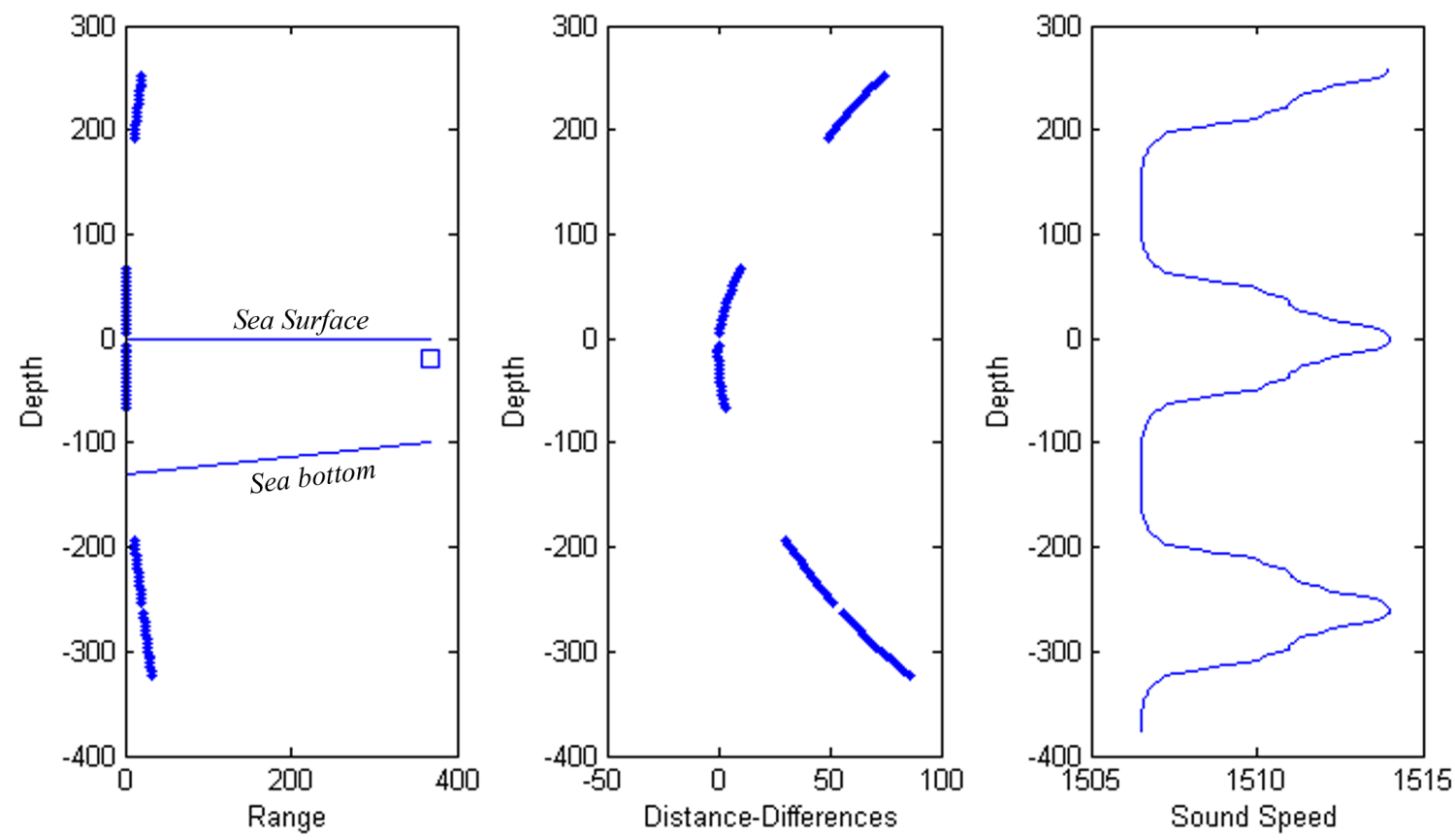

Fig. 4. (a) Physical array and its surface, bottom, surface-bottom and bottom-surface reflections with sloped bathymetry. The source is represented by a square on the right side. (b) Range differences to each hydrophone, relative to the source-to-reference range (topmost physical hydrophone). (c) Expanded sound speed profile for surface, bottom, surface-bottom and bottom-surface reflections.

and then recomputing those coordinates. Our results suggest that only two CSL iterations are enough to reach a solution with sufficiently good quality to initialize the model-based localization scheme described in Section V.

In general, the length of a curve between two abscissae $a$ and $b$ is given by the line integral

$$
l=\int_{a}^{b} \sqrt{1+\left(\frac{d f}{d x}\right)^{2}} d x .
$$

Using (8) we can derive the following expression for computing acoustic ray lengths between an acoustic source at depth $z_{s}$ and a hydrophone at depth $z_{m}$

$$
l=\int_{z_{s}}^{z_{m}} \sqrt{\frac{1}{1-(\rho c(z))^{2}}} d z,
$$

where $\rho$ denotes the ray parameter, which remains constant along the ray and is related to the grazing angle, $\theta$, as [1], [14]

$$
\rho=\frac{\cos \theta(z)}{c(z)} .
$$

The problem of determining the ray parameters of eigenrays linking the source and each hydrophone (or, equivalently, a single eigenray linking the source and each hydrophone image) is coupled with determining the ray trajectories themselves. An iterative scheme described in [14] can be used to efficiently compute eigenrays, starting with initial straight estimates connecting the acoustic source and the receivers. Alternatively, an acoustic simulator based on ray tracing can be used to compute eigenrays, from which the ray parameters are readily obtained from (10) based on the known departure or arrival angles and depths.

When $z_{s} \approx z_{m}$, so that $\theta(z) \approx 0$, we face a problem of numerical ill-conditioning in (9) trying to integrate a function with large values over a small interval. A change of variables can be used to express (9) as an integral between the source and receiver ranges with an integrand function taking on smaller values

$$
l=\int_{r_{s}}^{r_{m}} \frac{1}{\rho c(z(r))} d r .
$$

Compared to (9), the numerical properties of (11) are much more favorable for the predominantly horizontal propagation scenarios of interest to our work.

Once the ray length $l$ and the Euclidean distance $r=$ $\left\|\mathbf{x}_{s}-\mathbf{s}_{m}\right\|$ are available we compute the scaled TDOA as $\tau_{m}^{\prime}=\tau_{m} \frac{r}{l}$ to be used in the CSL algorithm. This compensation procedure is more effective at ranges on the order of 1 $\mathrm{km}$ and higher, where ray bending becomes significant.

\section{Model-Based Wavefront Matching}

The final processing step refines the source position, previously estimated by CSL, using a realistic model-based approach to match the predicted vs. observed pattern of wavefronts impinging upon the array. The method is conceptually similar to the one proposed in [15] to match the direct path and surface reflection, linearizing the propagation delays to attain a quadratic cost function that is easy to minimize. However, we 
consider more wavefronts (Direct, Surface, Bottom, SurfaceBottom and Bottom Surface paths) than the two used in [15], which effectively increases the spatial aperture of the array and leads to better estimation accuracy by improving the shape of the cost function at the minimum point.

Our cost function penalizes the Euclidean norm of predicted vs. observed TDOA differences for all hydrophones and wavefronts. Specifically, we iteratively solve

$$
\min _{\mathbf{x}_{s}} f\left(\mathbf{x}_{s}\right)=\left\|\boldsymbol{\tau}-\hat{\boldsymbol{\tau}}\left(\mathbf{x}_{s}\right)\right\|^{2},
$$

where $\tau$ denotes the vector of actual TDOAs, obtained by segmenting and classifying the observed wavefronts as described in Section III, and $\hat{\boldsymbol{\tau}}\left(\mathrm{x}_{s}\right)$ is the corresponding vector of TDOAs obtained from the acoustic ray tracer for a source located at $\mathbf{x}_{s}$.

To iteratively solve the nonlinear least-squares problem defined in (12) we need to compute partial derivatives of time delays with respect to acoustic source depth, $z_{s}$, and range, $r_{s}$. We use the following results from [14]

$$
\frac{\partial \tau}{\partial z_{s}}=\frac{\sqrt{1-\left(\rho c\left(z_{s}\right)\right)^{2}}}{c\left(z_{s}\right)}, \quad \frac{\partial \tau}{\partial r_{s}}=\rho,
$$

to obtain a first-order approximation of $\hat{\boldsymbol{\tau}}\left(\mathbf{x}_{s}\right)$ in (12) around the current source position estimate $\mathbf{x}_{s, k}$ as

$$
\hat{\boldsymbol{\tau}}_{k}\left(\mathbf{x}_{s}\right) \triangleq \hat{\boldsymbol{\tau}}\left(\mathbf{x}_{s, k}\right)+D \hat{\boldsymbol{\tau}}\left(\mathbf{x}_{s, k}\right)\left(\mathbf{x}_{s}-\mathbf{x}_{s, k}\right),
$$

where $D \hat{\boldsymbol{\tau}}\left(\mathbf{x}_{s, k}\right)$ denotes the Jacobian matrix. For $M$ sensors and $N$ wavefronts $\hat{\boldsymbol{\tau}} \in \mathbb{R}^{M N}$ and $D \hat{\boldsymbol{\tau}} \in \mathbb{R}^{M N \times 2}$. In each iteration of the minimization algorithm we update the current source position estimate by solving the linear LS problem

$$
\mathbf{x}_{s, k+1}=\arg \min _{\mathbf{x}_{s}}\left\|\boldsymbol{\tau}-\hat{\boldsymbol{\tau}}_{k}\left(\mathbf{x}_{s}\right)\right\|^{2} .
$$

Note that (15) resembles a maximization-minimization (MM) iteration for (12) [16], although the norm in (15) does not constitute a true majorization function for the original $f\left(\mathbf{x}_{s}\right)$ at point $\mathbf{x}_{s, k}$. Despite the lack of convergence guarantees, in practice we have observed fast and robust convergence of (15).

The full iterative source localization procedure is as follows:

1) The UWA channel response is estimated using basis pursuit method described in Section II.

2) Using wavefront segmentation methods from Section III, the TDOA for each hydrophone and path is computed (relative to the first arrival on the topmost hydrophone).

3) The initial guess for source position to be used in MBSL, $\mathbf{x}_{s, 0}$, is obtained by implementing the CSL scheme of Section IV.

4) An acoustic channel model is used to predict the time delays for the current source location estimate.

5) By computing the derivatives of time delay with respect to the acoustic source depth and communication range from (13), we linearize the delay function according to (14) and obtain an updated source position by solving the overdetermined linear LS problem (15).

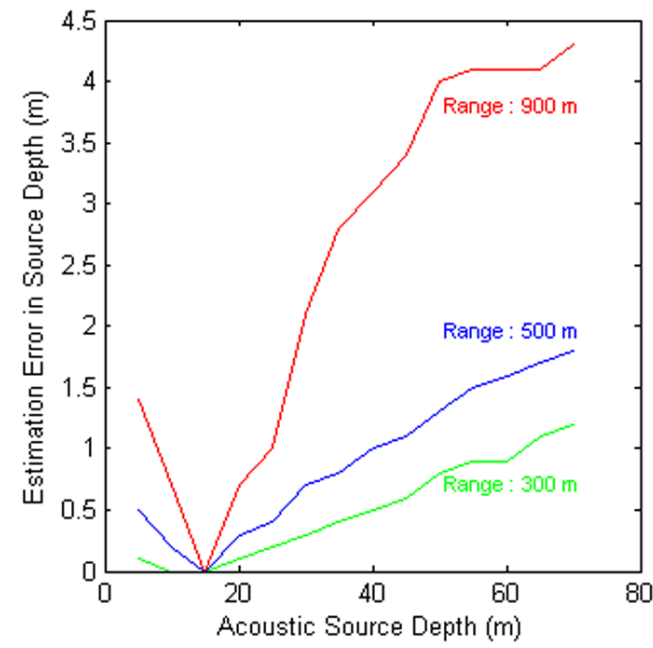

(a)

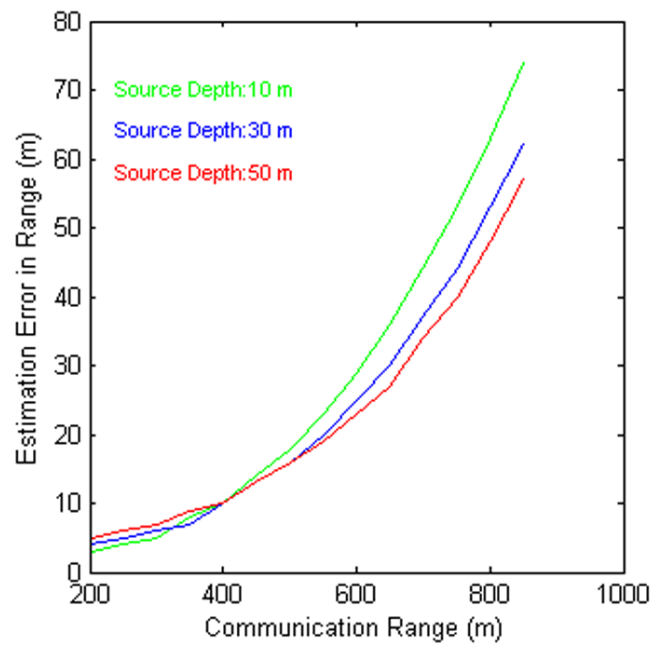

(b)

Fig. 5. Simulation results for localization using CSL with flat bathymetry, $120 \mathrm{~m}$ depth. Hydrophones are placed at depths of $6 \mathrm{~m}$ to $66 \mathrm{~m}$ with $4 \mathrm{~m}$ spacing. (a) Estimation error in source depth for different ranges. (b) Range estimation error for different source depths.

6) Check whether the solution satisfies a stopping criterion (e.g., marginal improvement in cost function or negligible change in estimated source position relative to the previous iteration) and go back to step 4 if necessary. Otherwise, the estimated source position is the final output an the procedure is over.

\section{PERformance Assessment}

In this section we evaluate the performances of CSL and MBSL where time delays are extracted from QPSK packet transmissions over simulated and real UWA channels.

\section{A. Simulation Results}

Simulation results were obtained using an online UWA simulator developed by the University of Algarve ${ }^{1}$. The

\footnotetext{
${ }^{1}$ http://www.ua-net.eu/projects/simulator/
} 


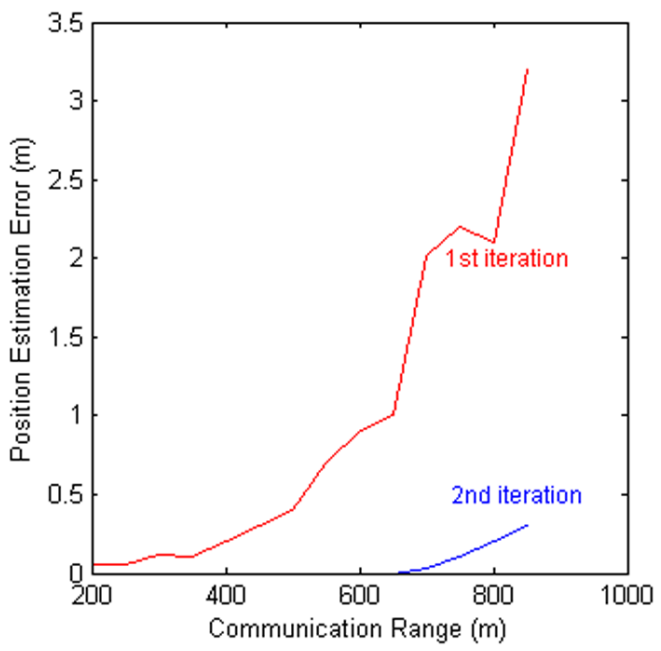

Fig. 6. Simulation results for localization using MBSL with flat bathymetry, $120 \mathrm{~m}$ depth. The plot shows the norm of the error vector between the estimated and actual source positions.

transmitted signal is a QPSK packet at 2.4 kbaud, with 5.5 $\mathrm{kHz}$ carrier frequency, $4.5 \mathrm{kHz}$ bandwidth, root-raised-cosine (RRC) pulse shape (88\% rolloff), and total duration $1 \mathrm{~s}$. The baseband received signal is sampled at 4 times the symbol rate, $f_{s}=9.6 \mathrm{kHz}$. The bottom is sandy $\left(1600 \mathrm{~m} / \mathrm{s}, 2 \mathrm{~g} / \mathrm{cm}^{3}, 0.8\right.$ $\mathrm{dB} / \lambda$ ) and located at $120 \mathrm{~m}$ depth. A total of 16 hydrophones are placed at $4 \mathrm{~m}$ intervals between $6 \mathrm{~m}$ and $66 \mathrm{~m}$ depths. DDSFs are estimated using TwIST [9].

Figure 5a shows the errors of CSL in estimating the depth of the acoustic source for three different horizontal ranges (300 $\mathrm{m}, 500 \mathrm{~m}$ and $900 \mathrm{~m}$ ), as a function of the actual source depth (varying from $5 \mathrm{~m}$ to $70 \mathrm{~m}$ ). While the three error curves go through zero for a source depth of about $15 \mathrm{~m}$, it is clear that, in general, larger depth estimation errors are obtained for longer communication ranges.

Figure 5b shows similar results for range errors at three different source depths as a function of the actual source range (varying from 200 to $850 \mathrm{~m}$ ). Unlike in Figure 5a, here no obvious correlation seems to exist between the magnitude of errors and the source depth. However, it is also clear that errors increase with communication range. This is to be expected, as the set of five selected wavefronts carry less information about the source position as it moves further into the far-field of the (expanded) receiver array. This more seriously affects range estimates than depth estimates (range errors are one order of magnitude larger), as the latter are computed along spatial directions that better exploit the array directivity, thus leading to less ambiguity.

Figure 6 shows the norm of the source localization error obtained by MBSL, with CSL initialization, as a function of range (source depth is $30 \mathrm{~m}$ ) after the first and second iterations. As in CSL, errors grow larger as the communication range increases. However, given the good initial point, MBSL is able to drastically reduce the total source localization error within 2 iterations. Note that there is no environmental

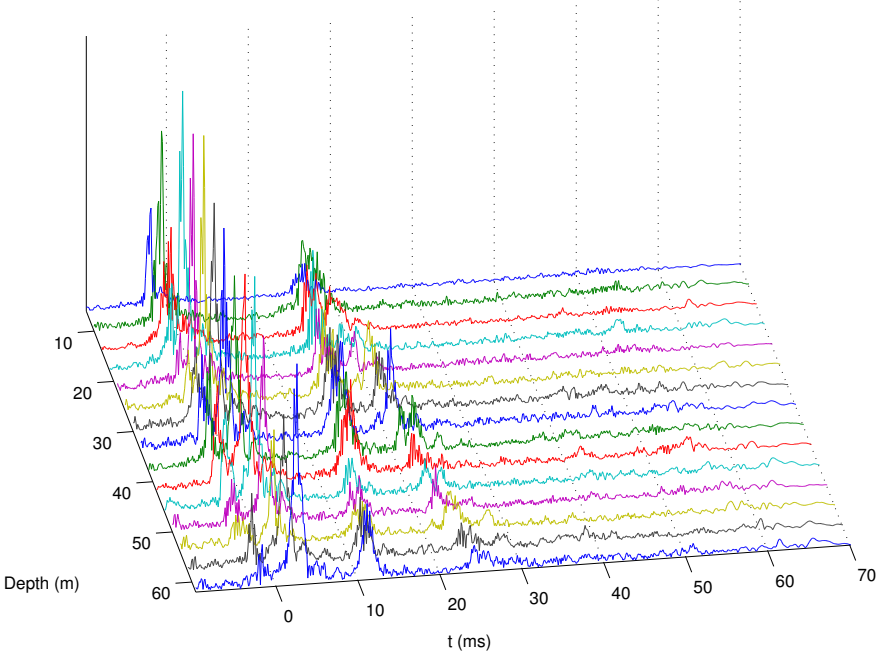

Fig. 7. Sample channel response for CALCOM'10, showing strong direct and surface reflected paths, and weaker bottom-interacting bounces.

mismatch in the ray tracer between data generation and computation of predicted delays.

\section{B. Experimental Results}

The CALCOM'10 sea trial was conducted south of Faro, Portugal, on June 22-24, 2010. The test area was approximately $3 \times 3 \mathrm{~km}^{2}$ at $36^{\circ} 52^{\prime} \mathrm{N}, 8^{\circ} 3.8^{\prime} \mathrm{W}$, with bottom sloping along the NE/SW direction from 100 to $200 \mathrm{~m}$. Bottom sediments at the site are silty, with an estimated compressional sound propagation speed of $1550 \mathrm{~m} / \mathrm{s}$. The SSP in the water column is downward refracting, decreasing from $1514 \mathrm{~m} / \mathrm{s}$ at the surface to $1507 \mathrm{~m} / \mathrm{s}$ at $60 \mathrm{~m}$, and remaining approximately constant below that. The receiver was a vertical drifting array with 16 uniformly-spaced hydrophones from $6 \mathrm{~m}$ to $66 \mathrm{~m}$ depth. Communication ranges during the experiment varied from about $300 \mathrm{~m}$ to $3 \mathrm{~km}$. The signals analyzed here were transmitted using a Lubell LL-1424HP source at $10 \mathrm{~m}$ depth.

We focus on QPSK packets at $5.6 \mathrm{kbit} / \mathrm{s}$, with $4.5 \mathrm{kHz}$ bandwidth, $5.5 \mathrm{kHz}$ carrier frequency, root-raised cosine pulse shapes (60\% rolloff), and total duration 3 s. Each packet is flanked by a pair of start/stop LFM markers for packet synchronization and coarse Doppler compensation. The arrival wavefront structure at the array is clearly visible, with strong direct and surface reflected paths, and weaker bottom and bottom-surface bounces. Figure 7 shows a sample response.

The estimation errors of CSL and MBSL for packets transmitted at different ranges are shown in figure 8. Rather than representing the norm of source position errors vectors, which would not take into account GPS errors and other positioning errors in our ground truth source location, we chose to compute the normalized norm of the difference between measured (i.e., segmented) and predicted TDOA values $\frac{1}{M N}\left\|\boldsymbol{\tau}-\hat{\boldsymbol{\tau}}\left(\mathbf{x}_{s}\right)\right\|$ (see (12)-(15) in Section V). From the results we see that MBSL significantly improves upon the CSL solution, even though the latter is already quite accurate. As expected, the estimation error decreases in the second 
iteration of MBSL, relative to the first one.

\section{CONCLUSION}

A two-step UWA source localization approach was proposed in this paper. In line with our previous work [8], this is intended as a contribution to the development of high-frequency tomographic methods that can be used to extract useful environmental information from communication signals, in addition to the digital messages themselves.

We have tested our algorithms in simulation for various source ranges from $200 \mathrm{~m}$ to $1 \mathrm{~km}$ and depths from $5 \mathrm{~m}$ to $70 \mathrm{~m}$. The paper also includes performance results using real data collected during the CALCOM'10 sea trial.

The first part of this scheme, termed "coarse source localization", tries to provide a good initial point for source position to be used for the second step, "model based source localization". The results presented in the paper demonstrate that the proposed CSL method is quite accurate and can be employed as a stand-alone estimator over short communication ranges (say, below $500 \mathrm{~m}$ ), or when only a rough source position estimate is needed. The fact that it dispenses with prior knowledge of the source location, and requires only crude environmental knowledge in its simplest form, is very appealing. In order to have a more accurate estimation when reliable environmental information is available, especially for longer communication ranges, the MBSL method can be used. In simulation MBSL converged to within $1 \mathrm{~m}$ of the true source position in as few as 2 iterations. However, MBSL is more computationally complex than CSL, as it involves running a ray trace at each iteration.

Future work will focus on streamlining and improving the accuracy of CSL. This could be done by improving the ray length computing block, so as to find robust ways to compute the ray length without using a simulator. Another relevant topic is to study the sensitivity of MBSL to environmental mismatch, and evaluate alternative iterative algorithms besides the linearized LS considered in [15], such as the Newtontype BFGS [17]. We will also pursue the estimation of source speeds in cases where we have moving sources, by including observed Doppler shifts in our model-based techniques.

\section{ACKNOWLEDGMENT}

This work was supported by Fundação para a Ciência e a Tecnologia through project PTDC/EEA-TEL/71263/2006 (PHITOM) and ISR/IST plurianual funding.

\section{REFERENCES}

[1] L. M. Brekhovskikh and Y. P. Lysanov, Fundamentals of Ocean Acoustics. Springer, 1991.

[2] F. B. Jensen, W. A. Kuperman, M. Porter, and H. Schmidt, Computational Ocean Acoustics. New York: American Institute of Physics Press, 1994.

[3] P. Hursky, M. B. Porter, M. Siderius, and V. K. McDonald, "Highfrequency (8-16 khz) model-based source localization," Journal of the Acoustical Society of America, vol. 115, pp. 3021-3032, 2004.

[4] D. Green, "Recovering data and voice recorders following at-sea crashes," in Proceedings of MTS/IEEE OCEANS'10, Sidney, Australia, May 2010.

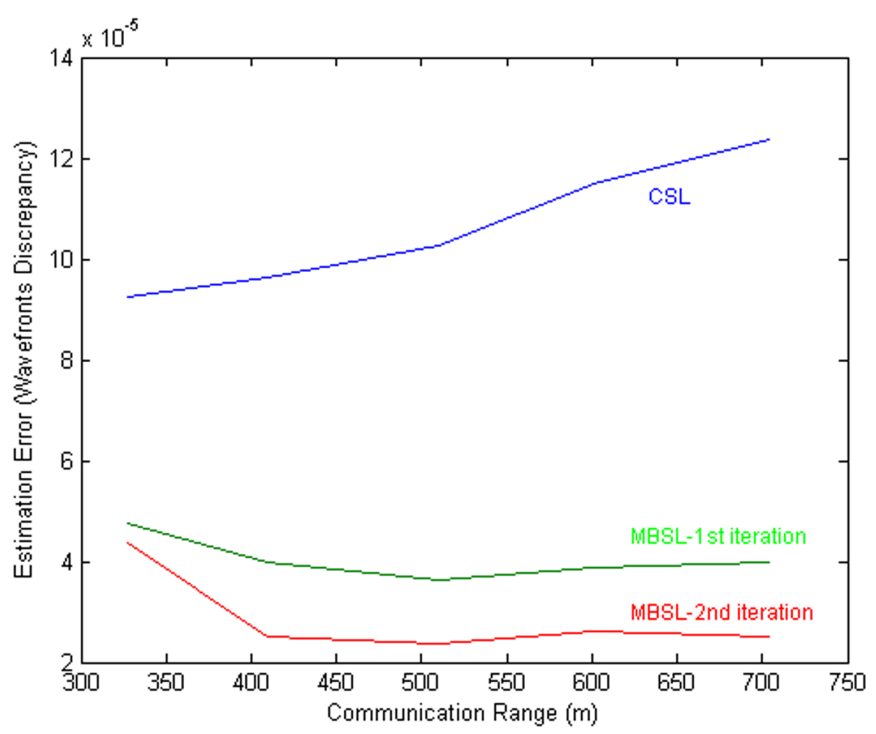

Fig. 8. Evaluation of CSL and MBSL methods with real CALCOM'10 data. The figure shows a global discrepancy metric of predicted vs. observed TDOAs.

[5] — "Beyond underwater acoustic communications," in Proceedings of MTS/IEEE OCEANS'11, Santander, Spain, June 2011.

[6] T. H. Eggen, A. B. Baggeroer, and J. C. Preisig, "Communication over Doppler spread channels - Part I: Channel and receiver presentation," IEEE Journal of Oceanic Engineering, vol. 25, no. 1, pp. 62-71, January 2000.

[7] P. A. Bello, "Characterization of randomly time-variant linear channels," IEEE Transactions on Communications Systems, vol. CS-11, pp. 360393, December 1963.

[8] E. Zamanizadeh, J. Gomes, and J. Bioucas-Dias, "Identification and matching of sparse delay-doppler spread functions from high-frequency communications signals," in OCEANS 2010, Seattle, Washington, USA, Sep 2010.

[9] J. M. Bioucas-Dias and M. A. T. Figueiredo, "A new TwIST: Twostep iterative shrinkage/thresholding algorithms for image restoration," IEEE Transactions on Image Processing, vol. 16, no. 2, pp. 2992-3004, December 2007.

[10] S. J. Wright, R. D. Nowak, and M. A. T. Figueiredo, "Sparse reconstruction by separable approximation," IEEE Transactions on Signal Processing, vol. 57, no. 7, pp. 2479-2493, July 2009.

[11] E. Zamanizadeh, J. Gomes, and J. Bioucas-Dias, "Identification of sparse time-varying underwater channels through basis pursuit methods," in Proceedings of the 10th European Conference on Underwater Acoustics (ECUA'10), Istanbul, Turkey, July 2010.

[12] J. Gomes, "Array processing methods for time-reversed underwater communication systems," Ph.D. dissertation, Instituto Superior Técnico, July 2002.

[13] A. Beck, P. Stoica, and J. Li, "Exact and approximate solutions of source localization problems," IEEE Transactions on Signal Processing, vol. 56, no. 5, pp. 1770-1778, 2008.

[14] S. E. Dosso, M. R. Fallat, B. J. Sotirin, and J. L. Newton, "Array element localization for horizontal arrays via occam's inversion," Journal of the Acoustical Society of America, vol. 104, pp. 846-859, 1998.

[15] X. Ma and Z. H. Michalopoulou, "Matched arrival processing for efficient inversion in underwater acoustics," in Proceedings of MTS/IEEE OCEANS'99, vol. 3, Seattle, USA, September 1999, pp. 1577-1580.

[16] D. Hunter and K. Lange, "A tutorial on MM algorithms," The American Statistician, vol. 58, no. 1, pp. 30-37, 2004.

[17] L. D. Hui and M. Fukushima, "A modified BFGS method and its global convergence in nonconvex minimization," Journal of Computational and Applied Mathematics, vol. 129, pp. 15-35, 2001. 